\title{
Estudo de 11 casos de fibroelastose endocárdica primária
}

\author{
Primary endocardial fibroelastosis: study of 11 cases
}

Lúcia de Noronha'; Fernando Augusto Fressato Hecke²; Tiago Augusto Magalhães; Diogo Lago Pinheiro4; Israil Cat ${ }^{5}$

unitermo
Fibroelastose
endocárdica

\section{resumo}

Introdução: A fibroelastose endocárdica (FEE) é definida como espessamento difuso do endocárdio ventricular em função da proliferação de fibras colágenas e elásticas. Pode ser classificada como primária ou secundária. A FEE primária apresenta-se na forma dilatada, mais comum, ou na forma restritiva. Geralmente manifesta-se como insuficiência cardíaca inexplicada, em crianças com menos de 12 meses de idade. Objetivos: Avaliar aspectos epidemiológicos, clínicos e anatomopatológicos da FEE primária. Material e método: Foram pesquisadas, entre 1960 e 2000, 6.211 necropsias perinatais e pediátricas do Hospital de Clínicas da Universidade Federal do Paraná (UFPR), tendo sido verificados 11 casos de FEE. Resultados: Em nove casos (81,8\%), o surgimento dos sintomas ocorreu antes de 1 ano de idade. Em oito $(72,7 \%)$, o óbito ocorreu antes de 1 ano de idade. Dentre os sinais clínicos, destacaram-se taquipnéia $(54,5 \%)$, hepatomegalia $(45,5 \%)$, tosse $(36,4 \%)$, cianose $(36,4 \%)$ e febre $(36,4 \%)$. Todas as radiografias $(n=6)$ apresentavam cardiomegalia. O espessamento endocárdico predominou no coração esquerdo $(90,9 \%)$. A causa de óbito foi insuficiência respiratória aguda em cinco casos $(45,5 \%)$ e choque cardiogênico em quatro (36,4\%). Discussão e conclusões: Os resultados mostram acometimento predominante de crianças com menos de 1 ano de idade e rápida progressão para óbito. Sinais clínicos freqüentes, como taquipnéia, tosse e febre, sugeriram infecção respiratória, que foi confirmada por necropsia em três casos (27,3\%). Os exames complementares mostraram apenas achados sugestivos de insuficiência cardíaca. Os dados analisados neste estudo mostram que, em nosso meio, a FEE primária não difere significativamente do que é apresentado na literatura internacional em relação a características epidemiológicas, clínicas e anatomopatológicas.

abstract

Background: Endocardial fibroelastosis (EFE) is a diffuse thickening of the ventricular endocardium resulting from proliferation of fibrous and elastic tissue. Primary and secondary forms have been described. Primary EFE occurs in the dilated and contracted forms. EFE usually manifests as unexplained congestive heart failure in infants younger than 1 year old. Objectives: To analyze the epidemiologic, clinical and anatomopathological aspects of primary EFE. Methods: Eleven cases of histologically confirmed EFE were found in 6211 necropsies performed between 1960 and 2000 in the Hospital of the University of Paraná. Results: In nine cases (81.8\%) the initial clinical presentation occurred before the age of 1 year. In eight cases (72.7\%) patients died before the age of 1 year. The most common clinical findings were tachypnea (54.5\%), hepatomegaly (45.5\%), cough (36.4\%) and fever (36.4\%). Cardiomegaly was the main radiographic finding, present in all cases $(n=6)$. The endocardial thickening was more frequent at the left heart (90.9\%). The death cause was acute respiratory failure in five cases (45.5\%) and cardiogenic chock in four cases (36.4\%). Discussion and conclusions: The results showed that EFE affects usually infants younger than 1 year old that rapidly progress to death. Some common clinical findings like tachypnea, cough and fever suggested respiratory infection which was confirmed by necropsy in three cases (27.3\%). The complementary tests showed only non-specific findings of congestive heart failure. The information analyzed here demonstrated no significant difference of the epidemiological, clinical and anatomopathological characteristics of primary EFE between Brazil and the international literature. key word

Endocardial fibroelastosis

1. Professora-adjunta da Disciplina de Patologia da Pontifíicia Universidade Católica do Paraná (PUCPR); doutora em Patologia.

2. Médico residente em Clínica Médica do Hospital Universitário Cajuru.

3. Médico residente em Radiologia da Aliança Saúde.

4. Acadêmico de Medicina; bolsista do Programa de Iniciação Cientítica (PIBIC)/PUCPR 2003/2004.

5. Professor-titular do Departamento de Pediatria da Universidade Federal do Paraná (UFPR).

Serviço de Anatomia Patológica (SAP) do Hospital de Clínicas (HC) da UFPR.

Laboratório de Patologia Experimental da PUCPR. 


\section{Introdução}

A fibroelastose endocárdica (FEE) é definida como espessamento difuso do endocárdio ventricular em razão da proliferação de fibras colágenas e elásticas $(3,4,15,18)$. A FEE comporta-se como uma miocardiopatia, em que o espessamento endocárdico leva à diminuição da complacência ventricular e à conseqüente insuficiência cardíaca ${ }^{(7,8,15)}$. Pode ser classificada como primária, quando não existem malformações cardíacas, ou secundária, quando uma malformação congênita do coração está presente. Malformações obstrutivas do ventrículo esquerdo, particularmente a estenose aórtica e a síndrome do coração esquerdo hipoplásico, estão freqüentemente associadas à $\mathrm{FEE}$ secundária(14, 18). A FEE primária pode se apresentar na forma dilatada, mais comum, ou na forma restritiva. Na forma dilatada, a cavidade ventricular esquerda apresenta-se aumentada; já na forma restritiva, está normal ou diminuída ${ }^{(1,5,6)}$.

lá foram levantadas diversas hipóteses sobre a etiologia da FEE primária, incluindo infecções virais, transmissão transplacentária de anticorpos, deficiência de carnitina e distúrbios metabólicos $(9,10,15,16)$. Já foram relatados casos de transmissão hereditária com padrões de transmissão autossômico recessivo e recessivo ligado ao $X^{(1,12,15,17)}$.

A FEE primária geralmente apresenta quadro clínico de insuficiência cardíaca congestiva inexplicada, em crianças com menos de 12 meses de idade ${ }^{(11)}$. A descompensação cardíaca é freqüentemente desencadeada por uma infecção respiratória ${ }^{(8)}$. A ecocardiografia pode sugerir o diagnóstico de FEE ao mostrar o endocárdio espessado, mas somente a biópsia endomiocárdica pode confirmá-lo ${ }^{(13)}$. O tratamento consiste no emprego precoce e prolongado de digitálicos e no transplante cardíaco ${ }^{(8)}$.

\section{Objetivos}

Este estudo tem o objetivo de avaliar os aspectos epidemiológicos, clínicos e anatomopatológicos da FEE primária e compará-los com a literatura pertinente.

\section{Material e método}

Foram realizadas, entre os anos de 1960 e 2000, 6.211 necropsias perinatais e pediátricas no Hospital de Clínicas da Universidade Federal do Paraná (UFPR). Entre todas as alterações cardiovasculares, foram encontrados 11 casos de FEE primária. O parâmetro utilizado para o diagnóstico de FEE primária foi o espessamento esbranquiçado e opaco do endocárdio à macroscopia e o espessamento fibroelástico do endocárdio à microscopia. $O$ critério de inclusão foi a presença de FEE confirmada pela histologia, na ausência de malformação cardíaca. Então, realizou-se estudo retrospectivo através da análise dos prontuários e relatórios de necropsia destes 11 pacientes, utilizando protocolo previamente estabelecido com os seguintes dados: sexo, idade ao surgimento dos sintomas cardiovasculares, idade quando do óbito, apresentação clínica inicial, exames complementares e dados da necropsia.

\section{Resultados}

Dos 11 casos estudados, 63,6\% $(n=7)$ eram do sexo masculino, com o intervalo de confiança de $95 \%$ de $28,4 \%$ a $92,1 \%$. Em nove casos ( $81,8 \%)$, o surgimento dos sintomas ocorreu antes de 1 ano de idade. Em oito casos (72,7\%), o óbito ocorreu antes de um ano de idade (Tabela 1). O intervalo entre o surgimento dos sintomas e o óbito foi menor que um mês em sete casos $(63,6 \%)$.

Entre as manifestações clínicas, as mais comuns foram taquipnéia (54,5\%), hepatomegalia (45,5\%), tosse (36,4\%), cianose $(36,4 \%)$, febre $(36,4 \%)$ e hipofonese de bulhas cardíacas $(36,4 \%)$. Outras manifestações menos comuns são listadas na Tabela 2. Nos exames complementares, todas as radiografias $(n=6)$ apresentaram cardiomegalia e houve um caso $(9,1 \%)$ com edema pulmonar. Entre as quatro eletrocardiografias disponíveis, taquicardia sinusal estava presente em $50 \%$, sobrecarga ventricular esquerda em $50 \%$, sobrecarga ventricular direita em $25 \%$, sobrecarga biventricular em $25 \%$, bloqueio atrioventricular de $1^{\circ}$ grau em $25 \%$, isquemia subepicárdica em $25 \%$ e isquemia subendocárdica em $25 \%$. A única ecocardiografia disponível mostrou dilatação de ventrículo esquerdo.

À necropsia, dez casos $(90,9 \%)$ apresentavam a forma dilatada e apenas um $(9,1 \%)$, a forma restritiva. Em seis casos (54,5\%), o espessamento endocárdico estava restrito ao ventrículo esquerdo e em dois casos $(18,2 \%)$, envolvia

Fibroelastose endocárdica primária: número de casos segundo a faixa etária no início dos sintomas e

Tabela 1 no óbito

$\begin{array}{lcc}\text { Faixa etária } & \text { Início dos sintomas } & \text { Óbito } \\ <1 \text { mês } & 4(36,4 \%) & 0(0 \%) \\ 1 \text { mês-1 ano } & 5(45,5 \%) & 8(72,7 \%) \\ >1 \text { ano } & 2(18,2 \%) & 3(27,3 \%)\end{array}$


Fibroelastose endocárdica primária: Tabela 2 manifestaçōes clínicas iniciais

\begin{tabular}{lc} 
Manifestações clínicas & $\begin{array}{c}\text { Número de casos } \\
\text { (percentual) }\end{array}$ \\
Taquipnéia & $6(54,5 \%)$ \\
Hepatomegalia & $5(45,5 \%)$ \\
Tosse & $4(45,5 \%)$ \\
Febre & $4(45,5 \%)$ \\
Cianose & $4(45,5 \%)$ \\
Hipofonese de bulhas & $4(45,5 \%)$ \\
Taquicardia & $3(27,3 \%)$ \\
Diarréia & $3(27,3 \%)$ \\
Vômitos & $3(27,3 \%)$ \\
$3^{a}$ bulha & $2(18,2 \%)$ \\
Estertores & $2(18,2 \%)$ \\
Gemência & $2(18,2 \%)$ \\
Sopro sistólico & $2(18,2 \%)$ \\
Jugulares ingurgitadas & $1(9,1 \%)$ \\
Edema generalizado & $1(9,1 \%)$ \\
\hline
\end{tabular}

átrio e ventrículo esquerdos. Em um caso $(9,1 \%)$, ambos ventrículos encontravam-se acometidos; em outro caso $(9,1 \%)$, o ventrículo direito e átrio direito; e, em outro $(9,1 \%)$, a emergência da aorta. Entre outros achados cardíacos da necropsia, destacam-se cardiomegalia, presente em todos os casos, hipertrofia de ventrículo esquerdo, em seis casos (54,5\%), endocardite bacteriana aguda com vegetação e destruição de valva mitral, em um caso $(9,1 \%)$, e insuficiência de valva mitral, em outro caso $(9,1 \%)$. Segundo os laudos de necropsia, a causa de óbito foi insuficiência respiratória aguda em cinco casos $(45,5 \%)$, choque cardiogênico em quatro casos $(36,4 \%)$, hipoglicemia em um caso $(9,1 \%)$ e anemia aguda em outro caso $(9,1 \%)$. Dentre os cinco casos de insuficiência respiratória aguda, três foram atribuídos a infecções respiratórias; um, a procedimento anestésico; e em outro caso não há especificação.

\section{Discussão e conclusões}

No presente estudo, não existiu predileção significativa da FEE pelo sexo masculino ou feminino. Assim como em estudos de Angelov et al., Ino et al. e Ni et al., a maior parte dos pacientes de nosso estudo $(81,8 \%)$ teve o surgimento de manifestações clínicas antes de completar o primeiro ano de idade. $O$ óbito ocorreu antes de 1 ano de idade em $72,7 \%$ dos casos, porcentual semelhante ao encontrado por $\mathrm{Ni}$ et al. (69\%) em estudo com 29 casos. Ni et al. observaram que nove dos 29 pacientes de seu estudo apresentaram intervalo de tempo inferior a um mês entre o aparecimento dos sintomas e o óbito (31\%), menos da metade do porcentual encontrado em nosso estudo (63,6\%). Nesse estudo de Ni et al, o surgimento dos sintomas cardiovasculares foi sempre observado antes dos 8 meses de idade, e a maior sobrevida foi de 7 anos. Com relação aos nossos dados, a sobrevida máxima foi de 2 anos de idade.

A apresentação clínica inicial mostrava principalmente manifestações que sugerem insuficiência cardíaca, como taquipnéia, hepatomegalia e cianose, e sinais que podem indicar infecção do trato respiratório, como febre e tosse. Friedman cita as infecções respiratórias como possível fator de descompensação cardíaca em pacientes com FEE. Apesar de os sinais clínicos citados não serem suficientemente específicos para afirmarmos que nossos pacientes possuíam infecção respiratória, a necropsia pode confirmar essa hipótese em três casos.

A insuficiência da valva mitral é complicação possível da FEE, em função da fibrose que envolve os músculos papilares $^{(8)}$. Em nossos pacientes, tivemos dois casos com sopro compatível com insuficiência mitral. A necropsia confirmou a presença de insuficiência mitral em um caso e mostrou endocardite bacteriana no outro.

Em estudo de Ino et al., a radiografia de tórax mostrou cardiomegalia em $99 \%$ dos pacientes com $\mathrm{FEE}$, fato que se repetiu em nossa amostra, em que o aumento da área cardíaca esteve presente em todas as radiografias. Outros achados radiográficos que podem estar presentes são aumento de átrio esquerdo e congestão pulmonar, sendo que o último pode ser demonstrado em um caso. Os achados eletrocardiográficos são inespecíficos e incluem principalmente sobrecarga de ventrículo esquerdo ${ }^{(11)}$, que foi demonstrada neste estudo em três dos quatro casos em que a eletrocardiografia estava disponível, incluindo um caso em que havia sobrecarga biventricular. No caso em que não existia sobrecarga ventricular esquerda, havia apenas sobrecarga de ventrículo direito, e a necropsia posteriormente mostrou que a doença estava restrita a átrio e ventrículo direitos. A ecocardiografia pode mostrar ecos densos ao longo do endocárdio ventricular esquerdo, achado bastante sugestivo de FEE, mas que Mahle et al. não consideram suficiente para indicar a estratégia a ser adotada. Na única ecocardiografia disponível neste estudo, a alteração que houve foi dilatação de ventrículo esquerdo. A única forma de se confirmar o diagnóstico é através da biópsia endomiocárdica ${ }^{(16,19)}$, que não foi realizada em nenhum dos pacientes deste estudo.

O acometimento predominante do coração esquerdo pela FEE (Figura), em especial o ventrículo esquerdo(1, 2, 4), 
é confirmado por este estudo, já que em apenas um caso $(9,1 \%)$, o coração esquerdo não foi acometido. O único caso deste estudo da forma restritiva, relativamente rara ${ }^{(6)}$, foi também o único caso que teve o espessamento endocárdico restrito ao coração direito. Entre as causas de óbito, destacaram-se a insuficiência respiratória aguda, presente em cinco casos $(45,5 \%)$ e que esteve presente em $71 \%$ dos pacientes do estudo de Ino et al., demonstrando a importância do possível papel de infecções respiratórias na desestabilização cardíaca nos pacientes com FEE.

Os dados apresentados neste estudo sugerem que a FEE primária, em nosso meio, não difere significativamente do que é apresentado na literatura internacional, no que diz respeito a características epidemiológicas, clínicas e anatomopatológicas.

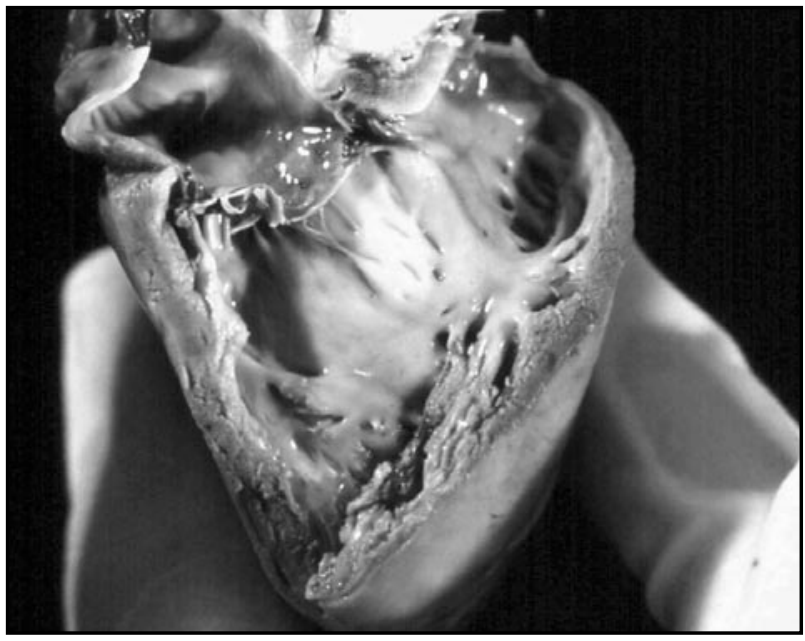

Figura - Ventrículo esquerdo e septo interventricular com espessamento esbranquiçado do endocárdio em caso de fibroelastose endocárdica primária

\section{Referências}

I. AKIBA, T. et al. Contracted form of endocardial fibroelastosis in two siblings. Pediat Int, v. 4I, n. 4, p. 379-8I, 1999.

2. ANGELOV, A.; KULOVA, A.; GURDEVSKY, M. Endocardial fibroelastosis. Clinico-pathological study of 38 cases. Pathol Res Pract, v. 178, n. 4, p. 384-8, 1984.

3. AROLA, A. et al. Idiopathic dilated cardiomyopathy in children: prognostic indicators and outcome. Pediatrics, v. I0I, n. 3, p. 396-76, 1998.

4. CABANERO, J. et al. Heart transplant in Ebstein's anomaly with endocardial fibroelastosis. Am Heart J, v. I 24, n. 2, p. 532-4, 1992.

5. CARVALHO,J.S.; Markiewicz, M. Dilated endocardial fibroelastosis: unusual late findings with congenital hydrops. Pediatr Cardiol, v. |8, p. 389-9|, 1997.

6. FARREL, D. J.; SKINNER, J. R. Restrictive endocardial fibroelastosis in a neonate without other cardiac pathology. J Clin Pathol, v. 45, p. 1042-3, 1992

7. FERENCZ, C.; NEILL, C. A. Cardiomyopathy in infancy: observations in an epidemiologic study. Pediatr Cardiol, v. 13, p. 65-7, 1992.

8. FRIEDMAN,W. F. Cardiopatias adquiridas na lactância e infância. In: BRAUNWALD, E. (ed.). Tratado de Medicina Cardiovascular. São Paulo: Roca, 1999. v.2, p. 1056.

9. GERSONY,W.M.; KATZ,S. L;, NADAS,A. S. Endocardial fibroelastosis and the mumps virus. Pediatrics, v. 37, n. 3, p. 430-4, 1966.

I0. HILTON, D. A.; VARIEND, S.; PRINGLE, J. H. Demonstration of Coxsackie virus RNA in formalin-fixed tissue sections from childhood myocarditis cases by in situ hybridization and the polymerase chain reaction.J Pathol, v. I70, p. 45-5I, 1993.

I I. INO,T.et al. Natural history and prognostic risk factors in endocardial fibroelastosis. Am J Cardiol, v. I 62, n. 7, p. 43 I-4, 1988

12. LEE, M. H. et al. Familial occurrence of endocardial fibroelastosis in three siblings, including identical twins. Pediatrics, v. 52 , n. 3, p. 402-।I, 1973
13. MAHLE,W.T.; Weinbweg, P. M.; Rychik, J. Can echocardiography predict the presence or absence of endocardial fibroelastosis in infants $<1$ year of age with left ventricular outflow obstruction? Am J Cardiol, v. 82, p. 122-4, 1998.

14. MIELKE, G. et al. Sequential development of fetal aortic valve stenosis and endocardial fibroelastosis during the second trimester of pregnancy. Am Heart J, v. 133, n. 5, p. 607-10, 1997.

15. NI, J. et al. Viral infection of the myocardium in endocardial fibroelastosis: molecular evidence for the role of mumps virus as an etiologic agent. Circulation, v. 95, n. I, p. 133-9, 1997.

16. NIELD, L. E. et al. Maternal anti-Ro and anti-La antibodyassociated endocardial fibroelastosis. Circulation, v. 105 n. 7, p. 843-8, 2002.

17. PYERITZ, R. E. Genetics and cardiovascular disease. In: BRAUNWALD, E. (ed.). Heart Disease. Philadelphia: W.B. Saunders, 2002. p. 1997.

18. WARD, C. J. et al. Angiographic demonstration of endocardial fibroelastosis in an infant with critical aortic stenosis. Am Heart J, v. 127, n. 5, p. 1430-I, 1994.

19. YOSHIZATO, T. et al. Safety and utility of endomyocardial biopsy in infants, children and adolescents: a review of 66 procedures in 53 patients. J Am Coll Cardiol, v. 15, n. 2, p. 443-5, 1990

Endereço para correspondência

Lúcia de Noronha Laboratório de Patologia Experimental Centro de Ciências Biológicas e da Saúde - Campus 1 Pontifícia Universidade Católica do Paraná Rua Imaculada Conceição 1.155 - Prado Velho CEP 80215-901 - Curitiba-PR Tel.: (41) 330-1515/ramal: 2264 Fax: (41) 330-1624 